\title{
External Quality Assessment Scheme for Transfusion Transmissible Infections among Blood Service Facilities in the Philippines, 2015
}

Rhoda Yu, Iza Mae Chamen, Kenneth Aristotle Punzalan, Benjamin de Vera III

Research Institute for Tropical Medicine

\section{ABSTRACT}

The External Quality Assessment Scheme (EQAS) for Blood Screening Serology aims to raise standards and assess the phases of laboratory testing of blood units.

In 2015, the National Blood Program listed a total of 200 Blood Service Facilities (BSF), 147 of which, enrolled for EQAS. These participants were given an EQAS panel designed to check the capacity of a BSF to detect the 5 transfusion transmitted infections (HIV, HBV, HCV, Syphilis and Malaria). Panels should be tested how a blood unit is routinely screened to mimic the actual laboratory process. This allows the NRL and participant to check and validate the entire blood unit screening process.

Test results were analyzed by OASYS Canada using the ISO 13528:2005 Robust Statistics method (Huber's Method) to identify outliers. Data analysis from the test event showed a significant number of participants that reported aberrant results due to errors related to random or systematic errors. This also showed deviations from standard practice recommended by the Department of Health as well as a comparison of different test platforms for blood screening.

Ultimately, the data gathered from the EQAS are used to improve on policies for blood screening and set recommendations for the safety of the Philippine blood supply.

Key words: blood transfusions, human immunodeficiency virus, hepatitis, syphilis, malaria, external quality assurance scheme, transfusion transmissible infections

\section{ISSN 0118-3265}

Printed in the Philippines.

Copyright (c) 2016 by the PJP.

Received: 13 March 2016.

Accepted: 21 April 2016.

Published online first: 27 April 2016.

http://dx.doi.org/10.21141/PJP.2016.005

Corresponding author: Kenneth Aristotle P. Punzalan, RMT

E-mail:tti.nr@@gail.com

\section{INTRODUCTION}

The EQAS for Blood Screening Serology provided by the Transfusion Transmissible Infections - National Reference Laboratory (TTI-NRL) of the Research Institute for Tropical Medicine aims to raise standards and assess the phases of laboratory testing on blood units to determine inter-laboratory comparison. The NRL-Australia cites the importance of EQAS as this provides objective evidence of quality through its capability to: (1) review kit and assay performance through monitoring consistency and accuracy of test results, (2) check on lab performance through comparison of different laboratory data, (3) identify random and systematic errors that needs to be managed, and (4) identification of laboratory's training needs. ${ }^{1}$

The EQAS panel is designed to check the capacity of a Blood Screening Facility (BSF) to detect the 5 common TTI. Samples of known reactivity to HIV, HBV, HCV, Syphilis and Malaria are to be tested in the same way as how a blood is routinely screened in the BSF as this mimics the routine samples received and screened. This allows the NRL and the participant to check and validate the blood unit screening process from receipt of samples up to release of results. As stated in DOH Department Circular No. 2013-0132, blood screening for TTI should only be done by licensed HIV proficient Medical Technologist and that all BSF are required to enroll for EQAS as per DOH Department Memorandum No. 2009-0086B. 


\section{METHODOLOGY}

\section{Panel Composition}

The serology EQAS panel for program code HVHT4320 consists of twenty (20) pooled plasma samples obtained from blood donors from different regions of the country. Each pooled sample was prepared by mixing similar volumes of at least two samples that had similar antibody and antigen profiles. All samples were subjected to filtration prior to aliquoting. The samples were aliquoted and their homogeneity confirmed. Representative samples were tested following shipment to participants to confirm their stability. The serology profile for HIV, HBV, HCV, Syphilis of each sample were identified using Chemiluminiscensce (ChLIA), Enzyme Immunoassay (EIA), Rapid Plasma Reagin (RPR), Particle Agglutination (PA) and Western Blot (WB).

Program code MLRA415 consists of five (5) blood smears. The samples were obtained from Malaria patients in Palawan and prepared by the NRL for Malaria and other Parasites of the Research Institute for Tropical Medicine.

\section{Participants}

The Multimarker Blood Serology EQAS panel ID HVHT4320 and Malaria Microscopy EQAS Panel ID MLRA415 were distributed to 147 participants nationwide. These participants enrolled for the EQAS 2015 Program with a corresponding registration fee to cover expenses for the test event.

Majority of the participants are private institutions followed closely by government institutions and the remainders are from the Philippine Red Cross. Figure 1 shows the distribution of BSF by region.

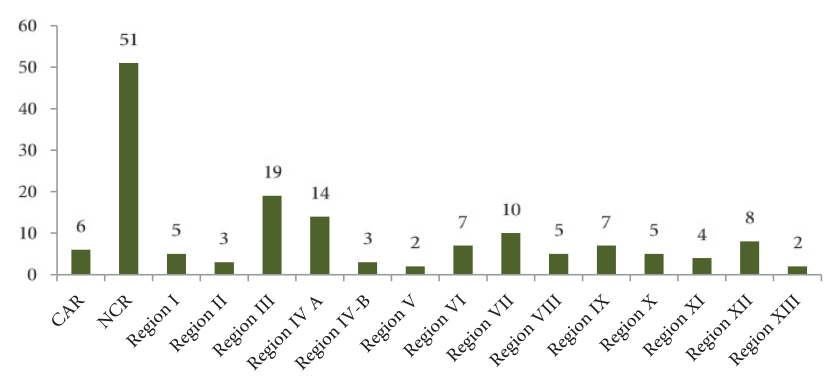

Figure 1. Regional distribution of participants.

\section{Data Analysis}

For data analysis, the TTI-NRL made use of the online informatics system (OASYS) developed and operated by Oneworld Accuracy Systems, Canada.

Participants were asked to enter assay results as well as assay interpretations in the online informatics system. Results reported by participating BSF for assay interpretations and final status were compared with the relevant reference results for qualitative evaluation. An assay interpretation that is different from the reference result is marked as aberrant.
The test results from the participating BSF were also sorted into peer groups. A peer group is defined as a set of laboratories that utilize the same test format and test kit/assay for screening TTI. The ISO 13528:2005 Robust Statistics method (Huber's Method) was used to identify outlying results (numerical test results found to be statistically different from other test results reported by participants that tested the same sample in the same assay) for the created peer groups. The said method uses the mean as an estimator. Outlying test results were removed from statistical calculation. ${ }^{2}$

\section{RESULTS AND DISCUSSION}

The predominant testing platform used by most participants was ChLIA followed by EIA, which is in concordance with what is being recommended by DOH DC No. 2013-0132. A significant number of participants are also using Rapid Diagnostic Test (RDT) kits for screening, which is not recommended for blood screening for the reason that these tests are often not as sensitive as EIA or instrument-based tests and can lead to false negative results in samples with low titres. ${ }^{3}$

One participant used an expired reagent for testing one analyte and 10 participants failed to indicate the expiry dates of their assay reagent kits. Six participants were identified to have reported results that were due to data entry error or clerical error (e.g. reactive test results were interpreted as negative or vice versa).

For the HVHT4320 serology panel, 19\% of 147 BSF reported aberrant results. Out of the 11,760 total number of results entered by the BSF, 11,722 (99.68\%) were correctly identified and $38(0.32 \%)$ were marked as aberrant. Out of the $0.32 \%$ aberrant results, 21 $(0.18 \%)$ and $17(0.14 \%)$ results were reported as false negative and false reactive respectively (Figure 2). Distribution of platform per TTI among aberrant results for the initial panel is shown in Table 1. These aberrant results were either due to data entry errors, sample mix-up or sample carry-over (particularly where an instrument was used in assay set-up).

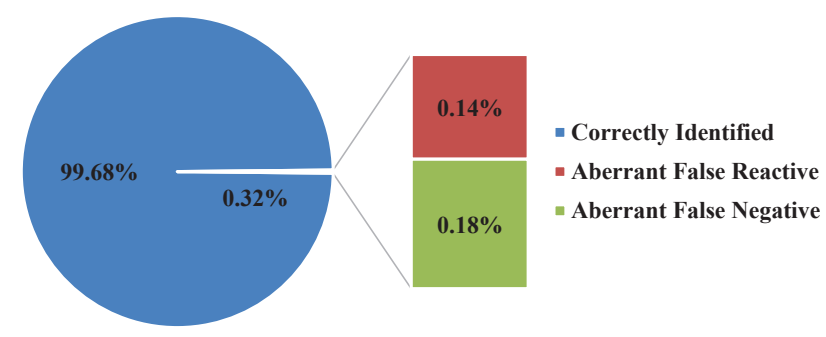

Figure 2. Percentage of aberrant results for HVHT4320 1st panel.

In testing the HVHT4320 initial panel, these criteria must be met for a BSF to be classified as having an unsatisfactory performance: (a) At least one false negative result; (b) At least twenty percent (20\%) false positive results. In accordance with these criteria, corresponding BSF, were given an investigation checklist to assist them in identifying their errors and make the necessary

\begin{tabular}{|c|c|c|c|c|c|c|c|c|c|}
\hline \multirow{2}{*}{ Platform } & \multicolumn{2}{|c|}{ HIV } & \multicolumn{2}{|c|}{ HBV } & \multicolumn{2}{|c|}{$\mathrm{HCV}$} & \multicolumn{2}{|c|}{ Syphilis } & \multirow{2}{*}{$\begin{array}{l}\text { Total } \\
\text { Aberrant }\end{array}$} \\
\hline & False Negative & False Reactive & False Negative & False Reactive & False Negative & False Reactive & False Negative & False Reactive & \\
\hline ChLIA & $3(7.89 \%)$ & $2(5.26 \%)$ & $3(7.89 \%)$ & $4(10.53 \%)$ & $2(5.26 \%)$ & $2(5.26 \%)$ & $0(0 \%)$ & $1(2.63 \%)$ & 17 (44.74\%) \\
\hline EIA & $1(2.63 \%)$ & $0(0 \%)$ & $1(2.63 \%)$ & $4(10.53 \%)$ & $2(5.26 \%)$ & $3(7.89 \%)$ & $1(2.63 \%)$ & $0(0.00 \%)$ & $12(31.58 \%)$ \\
\hline RDT & $0(0 \%)$ & $0(0 \%)$ & 5 (13.16\%) & $0(0 \%)$ & $0(0 \%)$ & $1(2.63 \%)$ & $2(5.26 \%)$ & $0(0 \%)$ & 8 (21.05\%) \\
\hline RPR & Not applicable & Not applicable & Not applicable & Not applicable & Not applicable & Not applicable & $1(2.63 \%)$ & $0(0 \%)$ & $1(2.63 \%)$ \\
\hline Total Aberrant & $4(10.53 \%)$ & $2(5.26 \%)$ & $9(23.68 \%)$ & $8(21.05 \%)$ & $4(10.53 \%)$ & $6(15.79 \%)$ & $4(10.53 \%)$ & $1(2.63 \%)$ & $38(100.00 \%)$ \\
\hline
\end{tabular}


corrective actions and/or troubleshooting methods. A 2nd set of the HVHT4320 panel were given to the BSFs for retesting if the identified unsatisfactory performance was due to a testing error. BSFs with aberrant results due to transcription errors were only given an investigation/troubleshooting checklist and a written recommendation. Five (5) BSFs were identified with transcription errors. Six (6) BSFs were given a second set of samples where only 3 were able to report each assay interpretation correctly.

Due to lack of accessibility to a good amount of inexpensive positive malaria blood samples, the NRL opted to provide a set of Blood Smears of known Malaria status to assess the capacity of the BSF to detect the presence of malaria parasite (qualitative identification only indicating presence or absence of the parasite). Although most, if not all, of the BSF perform platforms such as EIA and RDT for Malaria testing, it must be noted that according to DOH DC No. 2013-0132, a BSF should have the minimum capacity to detect the presence of malaria parasite through its gold standard, Malaria Microscopy. Presently, Malaria EIA kits in the country are neither evaluated nor regulated. The NRL for Malaria and other Parasites is in the process of evaluating these kits in partnership with the TTI-NRL. The microscopic diagnosis technique remains the gold standard for laboratory confirmation of malaria. ${ }^{4}$

For the MLRA415 panel, $12 \%$ of participants reported aberrant results and out of these, $9 \%$ reported false detection of human Plasmodia and $3 \%$ reported having false negative slides. This may be attributed to the fact that technicians are not proficient in reading malaria smears.

Figure 3 shows the distribution of grades of the BSFs. BSFs were evaluated and graded as follows:

- Excellent - 100\% acceptable results on the initial panel (all final results were correctly identified in comparison with the reference results);

- Very Satisfactory - Less than $100 \%$ acceptable results on the initial panel without being given a second panel for retesting. (A second panel is given to the BSF if upon comparison with the TTI-NRL reference result, there is at least one false negative or at least $20 \%$ false positive results reported);

- Satisfactory - $100 \%$ acceptable results on retesting of second panel (all final results are correctly identified in comparison with the reference result); or had an aberrant result in the initial panel due to clerical error (provided that the BSF identified the clerical error upon run through of the EQAS Investigation Checklist);

- Poor - BSF did not follow minimum requirements of testing as per DOH - DC 2013-0132 or; less than 100\% acceptable results on retesting of second panel (in comparison with the reference result, there is at least one false negative or at least $10 \%$ false positive results reported); or had an aberrant result in the initial panel that is due to clerical error which the BSF failed to identify upon run through of the EQAS Investigation Checklist.

According to DOH Memorandum 2009-0086B, EQA Participation and Proficiency testing with 2 or more consecutive failures, unsatisfactory, unacceptable results shall comply with the guidelines of the respective NRL. As an added quality assurance activity, the TTI-NRL conducts site-visits and assessment to BSF that attained satisfactory results and below. A detailed summary report and necessary recommendations are given to the BSF and the DOH for necessary actions.

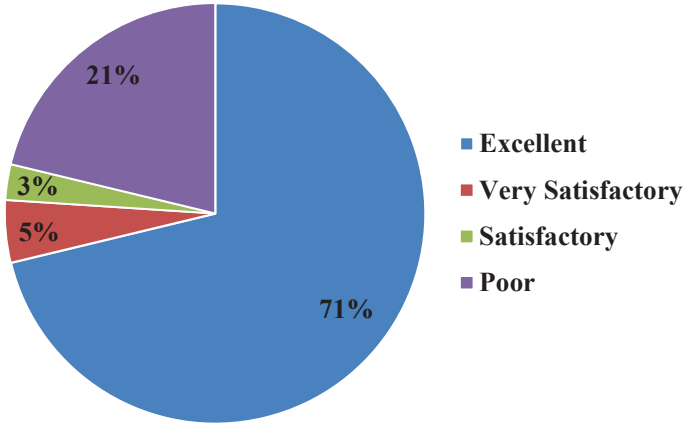

Figure 3. Distribution of grades for the EQAS 2015 test event.

\section{CONCLUSION}

Since the DC No. 2013-0132 was implemented in 2013, some BSFs still do not acknowledge or comply with the recommendations provided therein. Stringent measures should be enforced by each BSF for the safety of our national blood supply.

It is recommended that BSFs check and monitor their testing performance to identify aberrant results and perform appropriate corrective actions. The use of assay test kits evaluated by the STD/ AIDS Cooperative Central Laboratory (SACCL) and recommended by the National Blood Program, adherence to the manufacturer's protocols, strict internal quality control procedures and critical supervisory review are measures to avoid technical deficiencies. A second person should also check the assay results independently prior to reporting as this can resolve data entry errors.

The testing staff of the BSFs must be theoretically and technically proficient in testing for transfusion transmissible infections as this increases the competence most especially in correlation of test results as well as proper identification of Malaria parasites through intensive Malaria Microscopy Training and Proficiency Testing of Transfusion Transmissible Infections.

The use of RDT kits for blood screening is of inadequate sensitivity compared to Enzyme Immunoassay or instrument-based tests. This can lead to false negative results in samples with low levels of the transfusion transmissible infection. BSFs using two platforms for screening are encouraged not to retest samples on kits of low specificity/sensitivity (e.g. initial screening on EIA or CLIA and retesting on RDT).

The Blood Screening Serology EQAS plays a vital role in the improvement of efficiency of BSFs that ultimately improves the overall quality of the National Blood Program. Active participation of BSFs in this EQAS program will positively strengthen the quality of their service as there will always be room for improvement and development in this system. ${ }^{5}$

\section{AUTHOR DISCLOSURE}

The authors declared no conflicts of interest.

\section{ACKNOWLEDGEMENT}

The authors thank the TTI-NRL staff, the Dr. Catherine Masangkay and Dr. Socorro Lupisan of the Research Institute for Tropical Medicine (RITM), the Health Facility Development 
Bureau, the Department of Health - National Voluntary Blood Services Program, the National Council for Blood Services Technical Working Group, the Department of Parasitology (RITM), Philippine Red Cross - National Blood Center (Port Area), Asian Hospital and Medical Center and OneWorld Accuracy - Canada.

We also thank all participating Blood Service Facilities for their cooperation.

\section{REFERENCES}

1. Dimech W. External quality assessment schemes: their importance to blood screening laboratories. Retrieved from http://www.nrl.gov.au/CA25782200833499/All/68A20E750F F8B940CA257895001CED36/\$file/EQASTheirimportance tobloodscreeninglabs.pdf.

2. ISO 13528:2015: statistical methods for use in proficiency testing by interlaboratory comparisons

3. Smeh K. Final report multimarker blood screening serology (MMBS4320). NRL Australia: Panel ID 2015-11-11. 14 December 2015.
4. Centers for Disease Control and Prevention. Malaria diagnosis (U.S.) - Microscopy. 10 July 2015. Available from http://www. cdc.gov/malaria/diagnosis_treatment/microscopy.html.

5. World Health Organization. Screening donated blood for transfusion - transmissible infections: recommendations. Geneva: WHO Press, 2010.

6. Chaudhary R, Das SS, Ojha S, et al. The external quality assessment scheme: five years' experience as a participating laboratory. AsianJ Transfus Sci. 2010;4(1): 28-30. http://dx.doi. org/10.4103/0973-6247.59388. PMCID: PMC2847341.

7. Department of Health. DOH Department Circular No. 2013-0132: National Council for Blood Services - technical committee recommendations, strategies, methodologies and algorithms for testing blood units for transfusion transmissible infection. 19 March 2013. Retrieved from http://ti-nrl.com/ wp-content/uploads/2014/11/08-DC2013-0132.pdf.

8. Department of Health. DOH Department Memorandum No. 2009-0086B: Amendment to Department Memorandum No. 2009-0086-A entitled, "Implementation of external quality assessment program as regulatory requirement for licensing of clinicallaboratories."8September 2014. Retrievedfromhttp://tinrl.com/wp-content/uploads/2014/11/DM-2009-0086-B.pdf.

Disclaimer: This journal is OPEN ACCESS, providing immediate access to its content on the principle that making research freely available to the public supports a greater global exchange of knowledge. As a requirement for submission to the PJP, all authors have accomplished an AUTHOR FORM, which declares that the ICMJE criteria for authorship have been met by each author listed, that the article represents original material, has not been published, accepted for publication in other journals, or concurrently submitted to other journals, and that all funding and conflicts of interest have been declared. Consent forms have been secured for the publication of information about patients or cases; otherwise, authors have declared that all means have been exhausted for securing consent. 